\title{
Contaminación acústica en el transporte sanitario urgente por carretera
}

\section{Acoustic contamination in urgent medical transportation by road}

\author{
S. Ballesteros ${ }^{1}$, S. Lorrio ${ }^{1}$, I. Molina ${ }^{2}$, M. Áriz ${ }^{1}$
}

\section{RESUMEN}

Fundamento. Determinar los niveles de exposición a ruido durante el transporte sanitario urgente por carretera y describir la percepción de deterioro de la función auditiva en los trabajadores del sector y el empleo de mecanismos de protección acústica.

Metodología. Estudio observacional realizado en Bizkaia (España). Mediante dosimetrías sonométricas se registraron los valores instantáneos máximos de nivel sonoro $\left(\mathrm{L}_{\text {pico }}\right)$ y niveles continuos equivalentes $\left(\mathrm{L}_{\mathrm{eq}}\right)$ medidos en una ambulancia tipo de la red de emergencias. Paralelamente, se realizó una encuesta sobre una muestra representativa compuesta por 127 trabajadores en la que se exploró la percepción subjetiva de pérdida de audición y posibles factores asociados, además de otras cuestiones de interés desde la perspectiva de la prevención de riesgos laborales.

Resultados. Se registraron $\mathrm{L}_{\text {pico }}$ oscilantes entre 80 y $109 \mathrm{dBA}$ en función de la ubicación. Los niveles $\mathrm{L}_{\mathrm{eq}}$ medios evaluados durante 10 asistencias, desde la activación hasta la llegada al incidente y durante el traslado al hospital fueron de 79,5 dBA y 74,3 dBA $(p<0,001)$ respectivamente. El $36,2 \%$ de los trabajadores encuestados refirió deterioro de la audición en los últimos años. Las variables independientes asociadas a un mayor riesgo fueron la edad mayor a 45 años, antigüedad profesional superior a 15 años y sexo masculino. Las estrategias más empleadas para mitigar el ruido de las sirenas durante la emergencia fueron cerrar las ventanillas de la ambulancia y el uso del atenuador durante la noche.

Conclusiones. Los valores de las dosimetrías no exceden los valores críticos estipulados, pero se observa una alta prevalencia referida por los trabajadores de deterioro auditivo. Debe incidirse en la adopción de estrategias orientadas a disminuir el ruido y sus efectos en el transporte sanitario. Palabras clave. Ruido. Pérdida auditiva provocada por ruido. Servicios médicos de urgencia. Salud laboral.

\begin{abstract}
Background. To determine levels of exposure to noise during urgent medical transportation by road and to describe the perception of hearing function impairment in workers and the use of hearing protection devices.
\end{abstract}

Material and methods. An observational study was performed in Bizkaia (Spain). The instantaneous maximum noise levels $\left(\mathrm{L}_{\text {peak }}\right)$ and its continuous equivalents $\left(\mathrm{L}_{\mathrm{eq}}\right)$ were recorded through sonometric dosimetries, measured in a "sample ambulance" of the emergency network. In parallel, a survey was conducted on a representative sample of 127 workers in which self-reported hearing loss and associated factors were explored, in addition to other issues of concern from the perspective of prevention of occupational hazards.

Results. $\mathrm{L}_{\text {peak }}$ oscillating between 80 and $109 \mathrm{dBA}$ were recorded depending on the location. The average $\mathrm{L}_{\mathrm{eq}}$ levels measured over 10 emergency calls, from activation until arrival at the incident and during transport to the hospital, were of $79.5 \mathrm{dBA}$ and $74.3 \mathrm{dBA}(\mathrm{p}<0.001)$ respectively. Thirty-six point two percent of the surveyed workers spoke of hearing impairment in recent years. The independent variables associated with increased risk were: age over 45 years, professional seniority over 15 years and male gender. The strategies used to mitigate the siren's noise during the emergency were closing the windows of the ambulance and using the dimmer at night.

Conclusions. The values of the dosimetries do not exceed the critical values provided, but there is a high prevalence of hearing-impaired problems reported by the workers. Emphasis should be placed on the adoption of strategies to reduce noise and its effects on the ambulance service.

Key words. Noise. Noise-induced hearing loss. Emergency medical services. Occupational health.
1. Enfermeros. Bilbao SAMUR - Protección Civil. 2. Técnico en Emergencias. Ambulancias Euskadi.

Recepción: 5 de abril de 2012

Aceptación provisional: 4 de mayo de 2012

Aceptación definitiva: 7 de mayo de 2012

\section{Correspondencia:}

Sendoa Ballesteros Peña

Bilbao SAMUR - Protección Civil. Ayuntamiento de Bilbao. Parque de bomberos de Miribilla

C/ Juan Carlos Gortázar 3

48003 Bilbao (Bizkaia)

E-mail: sendoa.ballesteros@gmail.com 


\section{INTRODUCCIÓN}

El ruido es uno de los agentes físicos perniciosos más extendidos en el medio laboral, siendo definido como un sonido no agradable que origina sensaciones molestas e interfiere con la actividad humana. Se estima que en España afecta al 37\% de los trabajadores $^{1}$. Cuando el ruido implica riesgo o daño para las personas o para el desarrollo de sus actividades o cuando genera efectos negativos sobre el medio ambiente se habla de contaminación acústica ${ }^{2}$.

La evidencia científica ha dejado patente que altos niveles de ruido inciden de forma negativa en la salud tanto física como psíquica de quienes se exponen al mismo ${ }^{3-5}$. La pérdida de capacidad auditiva provocada por el ruido sigue siendo una de las enfermedades profesionales más comúnmente reconocida $\mathrm{y}$, a menudo, viene acompañada de tinnitus o zumbidos en los oídos. Además existe una marcada relación entre exposición laboral al ruido con modificaciones de la tensión arterial y frecuencia cardiaca ${ }^{6,7}$ y con la aparición de enfermedades cardiovasculares, además de con estrés o alteraciones digestivas ${ }^{5,8}$.

En el entorno sanitario, recientes investigaciones ${ }^{9}$ han determinado que incluso en los hospitales se exceden los niveles de ruido recomendados por la Organización Mundial de la Salud (establecidos, para esta situación, en 35 decibelios), siendo la contaminación sonora una queja frecuente, tanto de los usuarios como del personal sanitario. Además, este factor ha mostrado especial repercusión en el tiempo de recuperación de los pacientes.

Los niveles de exposición y riesgos asociados al ruido ha sido una temática ampliamente estudiada en diversos ambientes laborales, como en la industria o en la educación, sin embargo, son escasos los estudios referidos al entorno de las emergencias sanitarias, donde la exposición a las sirenas de los vehículos de urgencia puede considerarse como una importante fuente de ruido. Es por ello que, el presente estudio, tiene por objetivos determinar los niveles de exposición a ruido durante el transporte sanitario urgente por carretera y describir las actitudes y creencias de los profesionales sanitarios sobre la exposición al ruido ocupacional, la percepción de deterioro de la función auditiva y el empleo de mecanismos de protección acústica.

\section{METODOLOGÍA}

Se llevó a cabo un estudio exploratorio en ambulancias de transporte urgente, utilizando como muestra de conveniencia las unidades del servicio municipal de emergencias extrahospitalarias de Bilbao. Todas las unidades contaban con amplificadores electrónicos de $100 \mathrm{~W}$ de potencia, sin sistema automático de variación de nivel de emisión y con el megáfono situado sobre el techo de la ambulancia, inmediatamente detrás del puente de luces. Esta dotación técnica supone un sistema habitual con que se equipan las ambulancias de nuestro entorno.

Se realizaron mediciones instantáneas máximas de nivel sonoro $\left(\mathrm{L}_{\text {pico }}\right)$ y niveles sonoros continuos equivalentes $\left(\mathrm{L}_{\mathrm{eq}}\right)$, utilizando para ello un sonómetro integrador tipo II (modelo OMNI-SL-5868P), calibrado acorde a las indicaciones del fabricante antes de cada uso. Se seleccionaron las escalas de ponderación de frecuencias A y C para la determinación de los niveles sonoros máximos, la escala A para los continuos y un tiempo de respuesta lento (modo Slow) en ambas mediciones.

Los niveles sonoros instantáneos máximos (o pico) fueron obtenidos en una única sesión para cada tono del amplificador (Wail o señal de entrada, es decir, la primera posición; Yelp o señal de cruce, la segunda posición; y la señal ecológica) con y sin atenuador. El sonómetro fue colocado utilizando trípode en las siguientes posiciones:

1. A 7,5 metros frente a la parte delantera de la ambulancia y a una altura de 1,60 metros.

2. En la cabina de conducción, en el asiento central:

a) Con las ventanillas abiertas.

b) Con las ventanillas cerradas.

3. En la cabina asistencial, en el asiento lateral del asistente, con todas las ventanillas cerradas. 
El nivel sonoro continuo equivalente es el nivel de un ruido constante hipotético correspondiente a la misma cantidad de energía acústica que el ruido real considerado en un punto determinado durante un período de tiempo. Dadas las peculiaridades del trabajo en los servicios de emergencias médicas, la estrategia de medición de niveles sonoros continuos, o dosimetría, se basó en la tarea u operación ${ }^{10}$, siendo realizada durante 10 asistencias reales en días laborables de enero y febrero de 2012 en un rango horario comprendido entre las 9 y 21 horas. El intervalo de grabación de niveles de ruido fue predefinido para 10 segundos. Los sonómetros fueron asignados al responsable sanitario de la dotación y las mediciones se realizaron independientemente durante las siguientes operaciones:

1. ESTADO 3 (E3): en la cabina de conducción, desde la activación urgente de la unidad hasta la llegada al lugar de destino.

2. ESTADO 6 (E6): en la célula asistencial, desde el inicio del traslado hasta la llegada al servicio de urgencias hospitalario.

De forma paralela, se estructuró un cuestionario orientado a explorar la percepción subjetiva del ruido como problemática laboral entre los trabajadores del transporte sanitario urgente, además de para conocer el tipo y frecuencia con que utilizan estrategias dirigidas a mitigar sus efectos.

Se establecieron como criterios de inclusión en la encuesta a todos los trabajadores (técnicos, enfermeros y médicos) destinados en Unidades de Soporte Vital Básico y Avanzado integrados en el Sistema de Emergencias Médicas de Bizkaia que accedieron a participar. No se incluyeron en el estudio a los voluntarios del sector.

El tamaño de la muestra (obtenida por técnica aleatoria simple) fue estimado para un nivel de confianza del $95 \%$ y un margen de error de $\pm 7 \%$, bajo la hipótesis más desfavorable de máxima indeterminación $(\mathrm{p}=\mathrm{q}=0,5)$.

El cuestionario, previamente pilotado, estaba conformado por un apartado de variables sociolaborales (sexo, edad, categoría profesional y antigüedad laboral en el sector) y una serie de preguntas sobre percepciones, conocimientos y actitudes referidas a la contaminación sonora en su ámbito de trabajo:

- La primera pregunta estaba orientada a conocer la percepción subjetiva de afectación del trabajador por ruido en el desarrollo de su actividad, cuantificada mediante una escala de estimación descriptiva que abarcaba las opciones "nada" - "poco" - "bastante" - "mucho".

- En un segundo subapartado se propuso al encuestado a señalar la frecuencia con que adopta medidas de protección acústica durante su jornada laboral, utilizando una escala de estimación descriptiva (siendo los extremos "nunca" y "siempre").

- Finalmente se enunciaron una serie de preguntas con respuesta cerrada "sí" - "no".

- ¿Has percibido deterioro de la audición en los últimos años?

○ ¿Lo has consultado con algún especialista?

- ¿Te has sometido recientemente a algún control audiométrico?

- En los últimos doce meses, ¿se te ha practicado algún reconocimiento médico específico por exposición a ruido por parte de tu empresa?

- ¿Sueles presentar zumbido de oídos incluso cuando no hay sonido real?

- En tu empresa o centro de trabajo, ¿conoces si hay algún delegado de prevención de riesgos laborales?

- ¿Has recibido formación/información sobre los riesgos para tu salud y tu seguridad relacionados con la exposición al ruido derivado del desempeño de tu tarea profesional?

El cuestionario fue distribuido, entre los meses de enero y marzo de 2012, en doble formato: en papel y en soporte digital, utilizando para su difusión el correo electrónico. 
Para el análisis de datos se utilizó el programa Epi Info 3.5.1. Se calcularon índices de estadística descriptiva (proporciones con sus respectivos intervalos de confianza del 95\% y medias con su desviación típica) y en el análisis inferencial se utilizaron el test t de Student para el contraste de hipótesis, estableciendo significación estadística cuando $\mathrm{p}<0,05$.

La magnitud de la asociación a la variable efecto "deterioro autopercibido de la audición" en función de diferentes covariables se evaluó mediante el cálculo de la Odds Ratio.

\section{RESULTADOS}

\section{Resultados sonométricos}

Los niveles sonoros instantáneos máximos determinados para los sonidos de la sirena de la ambulancia oscilan en un rango de $80 \mathrm{dbA}$, obtenidos en la cabina de conducción con las ventanillas cerradas y el tono ecológico, y $109 \mathrm{dBA}$, a 7,5 metros frente a la unidad con el primer tono. Con la utilización del atenuador de potencia las mediciones descendieron hasta 67 y 93 dBA en las mismas localizaciones respectivamente (Tabla 1).

Tabla 1. Mediciones instantáneas máximas de nivel sonoro $L_{\text {pico }}$ (medidos en escalas A y C) de los tonos de la sirena en función de la ubicación

\begin{tabular}{lcccccc}
\hline & \multicolumn{3}{c}{ SIN ATENUADOR (dBA/dBC) } & \multicolumn{3}{c}{ CON ATENUADOR (dBA/dBC) } \\
\cline { 2 - 7 } & S1 & S2 & S3 & S1 & S2 & S3 \\
\hline Frente a la unidad & $109 / 109$ & $100 / 107,5$ & $96 / 104$ & $93 / 98,5$ & $87 / 95,5$ & $83 / 91$ \\
Cabina conducción & & & & & & \\
$\quad$ Ventanillas cerradas & $87 / 86$ & $84,5 / 84,5$ & $80 / 78,5$ & $74,5 / 76$ & $72,5 / 74$ & $67 / 72$ \\
$\quad$ Ventanillas abiertas & $102 / 95,5$ & $97,5 / 94,5$ & $87 / 87$ & $90 / 84,5$ & $86 / 84$ & $77 / 76,5$ \\
Cabina asistencial & & & & & & \\
$\quad$ Ventanilla cerrada & $95 / 88$ & $90 / 85,5$ & $83 / 78$ & $81 / 77$ & $78 / 74,5$ & $69 / 71$ \\
\hline
\end{tabular}

S1: Wail; S2: Yelp; S3: tono ecológico.

El nivel sonoro continuo equivalente durante los trayectos hasta el incidente en servicio de urgencia (ESTADO 3) tuvo un valor medio de 79,5 dBA (Dt 1) y de 74,3 dBA (Dt 1,9) durante los traslados hasta el centro hospitalario (ESTADO 6), hallándose diferencias significativas entre ambos $\left(\mathrm{t}_{(18)}=7,8 ; \mathrm{p}<0,001\right)$. La descripción pormenorizada de las mediciones realizadas en cada operación se representa en la tabla 2.

Tabla 2. Nivel sonoro continuo equivalente $\left(\mathrm{L}_{\mathrm{eq}}\right)$ y duración de la medición de la operación registrada en cada actuación real

\begin{tabular}{ccccc}
\hline & \multicolumn{2}{c}{ ESTADO 3 } & \multicolumn{2}{c}{ ESTADO 6 } \\
\hline Leq (dBA) & Duración (min: seg) & Leq (dBA) & Duración (min: seg) \\
\hline Medición 1 & 79,2 & $8: 00$ & 73,0 & $14: 16$ \\
Medición 2 & 79,2 & $5: 25$ & 73,2 & $8: 26$ \\
Medición 3 & 78,8 & $5: 40$ & 74,5 & $9: 16$ \\
Medición 4 & 79,3 & $4: 10$ & 71,7 & $9: 31$ \\
Medición 5 & 78,3 & $4: 16$ & 74,9 & $10: 51$ \\
Medición 6 & 80,4 & $3: 50$ & 76,4 & $9: 30$ \\
Medición 7 & 81,1 & $2: 10$ & 76,8 & $8: 45$ \\
Medición 8 & 78,2 & $9: 16$ & 76,9 & $4: 50$ \\
Medición 9 & 80,6 & $3: 50$ & 72,8 & $10: 31$ \\
Medición 10 & 80,1 & 73,2 & $6: 00$ \\
\hline
\end{tabular}

ESTADO 3: trayecto comprendido desde la activación de la unidad hasta la llegada al incidente.

ESTADO 6: trayecto desde el lugar del incidente hasta el Servicio de Urgencias Hospitalario. 


\section{Resultados del cuestionario}

Fueron encuestados 127 trabajadores del transporte sanitario urgente donde 82 ejercían mayoritariamente como conductores y 45 como sanitarios (técnicos, enfermeros o médicos). La edad y antigüedad profesional media en los participantes fue de 37,19 (Dt: 9,84) y 10,04 (Dt: 7,74) respectivamente, localizando diferencias significativas en estos valores al contrastarlos en función del sexo $\left(\mathrm{t}_{(125)}=3,875 ; \mathrm{p}<0,001 \mathrm{y}\right.$ $\left.\mathrm{t}_{(125)}=5,639 ; \mathrm{p}<0,001\right)$, resultando ambos superiores en el colectivo masculino.

El $66,14 \%$ (IC 95\% 57,21-74,30) de los encuestados refirió considerar el ruido como un riesgo asociado a su desempeño profesional. Sin embargo, un 50,39\% (IC 95\% 41,39-59,38) se mostró como poco o nada afectado por el ruido en tu actividad laboral, frente a un $36,22 \%$ (IC 95\% 27,88$45,22)$ que se calificó como bastante y un $11,81 \%$ (IC $95 \%$ 6,67-18,73) como muy afectado.
Reconoció un deterioro de la audición en los últimos años un 36,22\% (IC 95\% 27,8845,22 ) de los participantes, presentando este colectivo medias de edad y de antigüedad profesional significativamente superiores $\left(\mathrm{t}_{(125)}=4,833 ; \mathrm{p}<0,001 \mathrm{y} \mathrm{t}_{(125)}=3,252\right.$; $\mathrm{p}=0,002$ respectivamente) frente a los que respondieron negativamente.

Además, el 14,96\% (IC 95\% 9,25-22,37) de la muestra manifestó presentar ocasionalmente zumbido de oídos (acúfenos) incluso en ausencia de sonido real.

La figura 1 representa la frecuencia con que los trabajadores adoptan, durante la emergencia, distintas estrategias orientadas a mitigar el efecto del ruido de las sirenas de la ambulancia. El cierre de las ventanillas del vehículo y el uso del atenuador de potencia durante la noche han supuesto las medidas más habitualmente empleadas, en contraposición con el uso de tapones o cascos en los oídos o la utilización del atenuador durante el día.

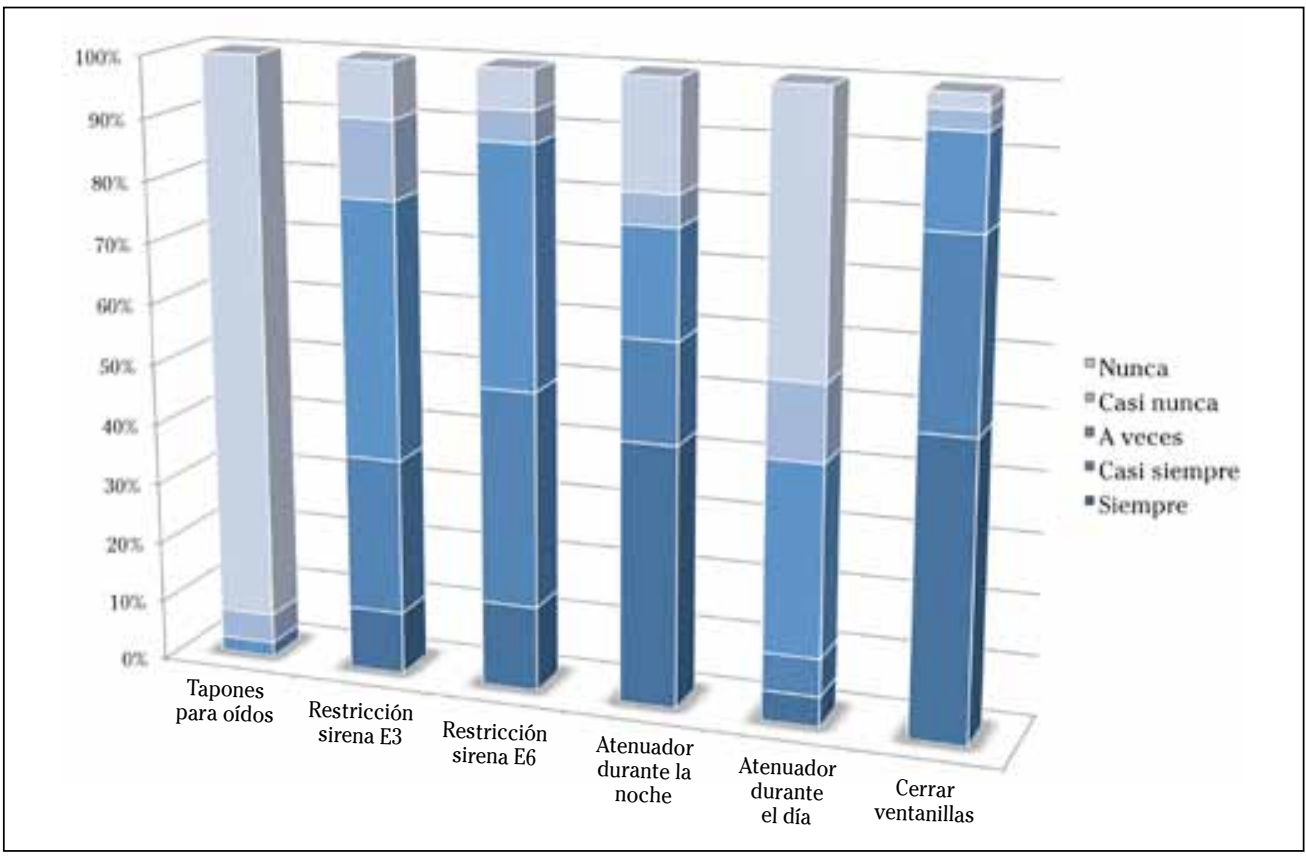

E3: Trayecto comprendido desde la activación de la unidad hasta la llegada al incidente; E6: trayecto desde el lugar del incidente hasta el Servicio de Urgencias Hospitalario.

Figura 1. Frecuencia con que los trabajadores adoptan distintas medidas o estrategias para mitigar el ruido durante el transporte sanitario por carretera. 
Tal y como se muestra en la Tabla 3 , la variable independiente más asociada a la percepción de deterioro de la audición fue la edad del trabajador superior a los 45 años. Antigüedades profesionales en el sector superior a 15 años y el sexo masculino también mostraron resultados significativos.

Tabla 3. Análisis de la asociación entre la deterioro autopercibido de la audición y diversas variables independientes

\begin{tabular}{|c|c|c|c|c|}
\hline & \multirow{2}{*}{$\begin{array}{c}\text { Total } \\
\text { n (\%; IC 95\%) }\end{array}$} & \multicolumn{2}{|c|}{ Deterioro de la audición } & \multirow{2}{*}{$\begin{array}{l}\text { OR cruda } \\
\text { (IC 95\%) }\end{array}$} \\
\hline & & Sí & NO & \\
\hline \multicolumn{5}{|l|}{ Sexo } \\
\hline Hombre & $\begin{array}{c}93 \\
(73,23 \% ; 64,65-80,69)\end{array}$ & 40 & 53 & $3,52(1,23-10,54)$ \\
\hline Mujer & $\begin{array}{c}34 \\
(26,77 \% ; 19,31-35,35)\end{array}$ & 6 & 28 & 1 (referencia) \\
\hline \multicolumn{5}{|l|}{ Perfil profesional } \\
\hline Sanitario & $\begin{array}{c}45 \\
(35,43 \% ; 27,15-44,41)\end{array}$ & 13 & 32 & $0,60(0,26-1,41)$ \\
\hline Conductor & $\begin{array}{c}82 \\
(64,57 \% ; 55,59-72,85)\end{array}$ & 33 & 49 & 1 (referencia) \\
\hline \multicolumn{5}{|l|}{ Edad } \\
\hline$>45$ años & $\begin{array}{c}31 \\
(24,41 \% ; 17,23-32,82)\end{array}$ & 20 & 11 & $9,09(1,43-73,68)$ \\
\hline 25-45 años & $\begin{array}{c}84 \\
(66,14 \% ; 57,21-74,30)\end{array}$ & 24 & 60 & $2,00(0,37-14,32)$ \\
\hline$<25$ años & $\begin{array}{c}12 \\
(9,45 \% ; 4,98-15,92)\end{array}$ & 2 & 10 & 1 (referencia) \\
\hline \multicolumn{5}{|c|}{ Antigüedad profesional en el sector } \\
\hline >15 años & $\begin{array}{c}28 \\
(22,05 \% ; 15,18-30,26)\end{array}$ & 17 & 11 & $5,22(1,54-18,29)$ \\
\hline 5-15 años & $\begin{array}{c}64 \\
(50,39 \% ; 41,39-59,38)\end{array}$ & 21 & 43 & $1,65(0,59-4,74)$ \\
\hline$<5$ años & $\begin{array}{c}35 \\
(27,56 \% ; 20,01-36,19)\end{array}$ & 8 & 27 & 1 (referencia) \\
\hline
\end{tabular}

Ha recibido formación/información sobre los riesgos relacionados con la exposición al ruido

$\begin{array}{lcccc}\text { Sí } & 28 & 6 & 22 & 0,40(0,13-1,17) \\ & (22,05 \% ; 15,18-30,26) & & & \\ \text { No } & 99 & 40 & 59 & 1 \text { (referencia) }\end{array}$

OR: odds ratio; IC 95\%: intervalo de confianza del $95 \%$

El 59,20\% (IC 95\% 50,05-67,90) de los consultados asintió haberse realizado recientemente algún control audiométrico y el $48,39 \%$ (IC 95\% 30,16-66,94) de quienes afirmaron haber percibido deterioro de la audición acudieron al médico especia- lista. Un 55,12\% (IC 95\% 46,04-63,95) de los profesionales declaró no conocer la existencia en su centro de trabajo de un delegado de Prevención de Riesgos Laborales y el $28,35 \%$ (IC $95 \%$ 20,71-37,02) notificó haberse sometido en el último año a 
un reconocimiento médico específico por exposición al ruido en el examen de salud de empresa.

\section{DISCUSIÓN}

Exposiciones prolongadas a ruido por debajo $80 \mathrm{dBA}$ no provocan alteraciones definitivas en el oído humano, sin embargo, no impide que puedan aparecer molestias pasajeras (fatiga auditiva). Cuando la intensidad supera los $90 \mathrm{dBA}$ aparecen lesiones irreversibles, que aumentarán cuanto mayor sea la exposición y la susceptibilidad individual ${ }^{10}$ : un ruido de $92 \mathrm{dBA}$ puede causar sordera a lo largo del tiempo si la exposición del trabajador excede tres horas diarias $^{8}$.

Además, debe tenerse en cuenta que el ruido puede producir efectos extraauditivos especialmente relevantes en la práctica clínica durante el transporte sanitario. A partir de $80 \mathrm{dBA}$ la actitud cooperativa se reduce, aumenta la agresividad ${ }^{11}$ y la inteligibilidad de una comunicación entre dos personas resulta dificultosa. De la misma manera, también se ha evidenciado que en exposiciones alrededor de los $82 \mathrm{dBA}$ los trabajadores tienen un riesgo 3 veces mayor de sufrir accidentes de trabajo ${ }^{12}$.

Las actuales normativas ${ }^{13}$ sobre la protección de los trabajadores frente al ruido establecen que a partir de niveles equivalentes diarios de $80 \mathrm{dBA}$ (o $\mathrm{L}_{\text {pico }}$ superior a $135 \mathrm{dBC}$ ) el uso de equipos de protección es recomendable, y obligatorio cuando supere los $85 \mathrm{dBA}$ (o $\mathrm{L}_{\text {pico }}$ superior a $137 \mathrm{dBC}$ ). Además, establece la justificación de la utilización de equipos de protección individual cuando los riesgos no se pueden evitar o no pueden limitarse suficientemente por otros medios técnicos.

$\mathrm{Si}$ bien los valores sonométricos determinados no se muestran como niveles equivalentes diarios (que variarán en función del número de asistencias realizadas a lo largo de la jornada) parece difícil que se lleguen a alcanzar cifras que obliguen a establecer un programa de medidas técnicoorganizativas destinado a disminuir el ruido. No obstante, las exposiciones próximas a los $80 \mathrm{dBA}$ registradas en algunas opera- ciones sí deberían hacer considerar la posibilidad de crear estrategias para mitigar al máximo la contaminación acústica generada en el transporte sanitario urgente.

Esta recomendación toma especial interés al constatar que alrededor del $36 \%$ de los trabajadores de la muestra estudiada refieren haber percibido un deterioro de la audición. El déficit auditivo inducido por ruido es un mal común que a menudo se subestima porque no provoca efectos inmediatos visibles ni se le otorga la consideración de "riesgo laboral"14. Esta pérdida puede ser tan gradual que pasa inadvertida hasta que el deterioro resulta discapacitante, situación que explicaría, al menos parcialmente, la mayor prevalencia referida en los trabajadores con más edad y/o antigüedad profesional. La hipoacusia neurosensorial representa la principal enfermedad relacionada con la exposición crónica a ruido de moderada-alta intensidad, causada por una lesión de los estereocilios del órgano de Corti, siendo únicamente eficaz la prevención, al no existir tratamiento curativo.

En la serie estudiada también se ha observado una prevalencia del deterioro de la audición tres veces superior en hombres frente a mujeres. No obstante, debe destacarse que el primer grupo también representa el colectivo de mayor edad y antigüedad profesional en el sector.

Cabría esperar que los trabajadores con perfil profesional sanitario (menos expuestos a los mayores niveles sonoros presentes en la cabina de conducción) o el hecho de haber recibido formación previa sobre los riesgos de la exposición laboral al ruido se asociasen a un menor deterioro de la audición, sin embrago, el análisis de asociación realizado no ha mostrado resultados estadísticamente significativos.

La publicación de investigaciones orientadas a la determinación de los niveles de ruido en el transporte sanitario por carretera y sus repercusiones sobre los trabajadores (o pacientes) son aún anecdóticos en la bibliografía, por lo que resulta difícil comparar los resultados obtenidos en nuestro estudio. Algunas reseñas de Estados Uni$\operatorname{dos}^{15,16}$ también refieren problemas de audición, presuntamente de etiología laboral, 
en paramédicos de ambulancias, con prevalencias de hasta un $15 \%$ de afectados. Contrariamente, otros trabajos ${ }^{17}$ no conciben el ruido como riesgo para la salud del personal de emergencias, notificando niveles sonométricos instantáneos máximos de 84 dBA en la cabina de conducción del vehículo con las ventanas abiertas. Esta última publicación contrasta notablemente con nuestros resultados, lo que debe orientarnos a pensar en la enorme variabilidad técnica de los vehículos destinados al transporte sanitario. Por ello, es preciso ser cauto a la hora de generalizar los resultados obtenidos a otras unidades, puesto que pueden ser influenciados por las particularidades, tanto de la ambulancia como del sistema organizativo del Servicio de Emergencias.

Una escasa concepción del ruido como riesgo laboral o sentimientos banales de su repercusión sobre la salud pueden motivar la no adopción de medidas preventivas orientadas a mitigar el impacto del ruido en el ámbito de trabajo. Tan sólo una pequeña proporción de los encuestados ha referido emplear, de manera regular, alguna estrategia de protección frente a la contaminación sonora generada en la atención extrahospitalaria de urgencias. El uso de cascos u otro tipo de protectores del oído es anecdótico en nuestro medio, mientras que en servicios de emergencias estadounidenses, la aplicación de esta medida ha sido constatada en más del $20 \%$ de los paramédicos ${ }^{16}$.

También se ha podido observar una mayor tendencia a restringir las señales acústicas durante los traslados al centro hospitalario, situación que explicaría los menores niveles sonoros continuos detectados en esta operación frente al ESTADO 3.

Por otro lado, y a pesar de ser habitual durante las horas nocturnas, el empleo del atenuador de potencia de la sirena durante el día resulta una acción poco extendida entre los conductores de vehículos de urgencia. Este sistema reduciría entre 12 y 16 dBA los niveles instantáneos máximos, colocando los valores sonométricos entre los marcados como autorizados para zonas urbanas, que en el caso de la Ordenanza Municipal de Bilbao de Protección del Medio Ambiente ${ }^{18}$ se sitúa en los 95 dBA (medidos a 7,5 metros en la dirección de máxima emisión). Esta norma es similar en otras áreas metropolitanas españolas, como en el caso de Madrid ${ }^{19}$, Huesca ${ }^{20}$ o A Coruña ${ }^{21}$.

Es preciso recordar que la escala de medida del sonido no es lineal sino logarítmica: un belio equivale a 10 decibelios y representa un aumento de potencia de 10 veces sobre la magnitud de referencia, 2 belios representan un aumento de 100 veces en la potencia, 3 belios equivalen a un aumento de 1000 veces y así sucesivamente (por ejemplo, un sonido con $86 \mathrm{~dB}$ tiene, el doble de fuerza que un sonido con $83 \mathrm{~dB}$ y 4 veces más que un sonido con $80 \mathrm{~dB}$ ).

La disposición para la prevención de lesiones ocasionadas por ruido en el ámbito de las emergencias extrahospitalarias no está demasiado arraigada en el área del transporte por carretera, como parece desprenderse de la discreta oferta o participación en controles audiométricos y la escasa información recibida por los trabajadores acerca de esta problemática.

La principal limitación a destacar en este trabajo radica en la ausencia de confirmaciones objetivas mediante análisis audiométricos de los deterioros sensoriales referidos por los profesionales. Sin embargo, creemos asumible esta condición dada la intencionalidad exploratoria de la investigación, incidiendo en la necesidad de desarrollar nuevos proyectos orientados a validar la hipótesis aquí planteada.

Con todo, es razonable concluir que, pese a no haber localizado en el medio que nos ocupa niveles de ruido que obliguen al suministro de equipos de protección específicos frente al ruido, sí debe ser prioritaria la adquisición de una conciencia de seguridad laboral y adopción de estrategias orientadas a la minimización de la contaminación acústica y de sus afectos, a la vista de un potencial problema de salud reportado por los trabajadores del sector.

\section{BIBLIOGRAFÍA}

1. Instituto Nacional de Seguridad e Higiene en el Trabajo. VI Encuesta Nacional de Condiciones de Trabajo. 2008. Ministerio de Trabajo y Asuntos Sociales. 
2. Ley $37 / 2003$, de 17 de noviembre, del Ruido. Boletín Oficial del Estado 276, 18 noviembre 2003.

3. Castells Murillo I. ¿Cómo nos afecta el ruido en nuestra salud, estilos de vida y entorno? ROL de Enferm 2007; 30 (10):13-20.

4. EdWARDS B. Cognitive and psicho social consequences of hearing loss. Audiology News 2009; 18: 96-98.

5. Cortés Barragán R, Maqueda Blasco J, Ordaz Castillo E, Asúnsolo del Barco A, Silva Mato A, Bermejo García E et al. Revisión sistemática y evidencia sobre exposición profesional a ruido y efectos extra-auditivos de naturaleza cardiovascular. Med Segur Trab 2009; 55 (215): 28-51.

6. Concha-Barrientos M. Occupational noise: assessing the burden of disease from workrelated hearing impairment at national and local levels. 2004. Geneve, World Health Organization. (WHO Environmental Burden of Disease Series, № 9).

7. Fogari R, Zoppi A, Vanasia A, Marasi G, Villa G. Occupational noise exposure and blood pressure. J Hypertens 1994; 12: 475-479.

8. Ganime JF, Almeida da Silva L, Robazzi ML do CC, Valenzuela Sauzo S, Faleiro SA. El ruido como riesgo laboral: una revisión de la literatura. Enfermería global 2010; 19: 1-15.

9. Yoder JC, Staisiunas PG, Meltzer DO, Knutson KL. Noise and sleep among adult medical inpatients: far from a quiet night. Arch Intern Med 2012; 172: 68-70.

10. Instituto Nacional de Seguridad e Higiene en el Trabajo. Guía técnica para la evaluación y prevención de los riesgos relacionados con la exposición de los trabajadores al ruido. Madrid: INSHT; 2008.

11. Oficina de defensor del ciudadano/a. No al ruido: guía de actuación. Normativas y vías de reclamación sobre ruido. Málaga: Oficina de defensor del ciudadano/a; 2010.

12. Ministerio de Ciencia e Innovación - Escuela Nacional de Medicina del Trabajo. Efectos extra-auditivos del ruido, salud, calidad de vida y rendimiento en el trabajo; actuación en vigilancia de la salud. Madrid, 2010.
13. Real Decreto 286/2006, de 10 de marzo, sobre la protección de la salud y la seguridad de los trabajadores contra los riesgos relacionados con la exposición al ruido. Boletín Oficial del Estado 60, 11 de Marzo de 2006.

14. García AM, Gadea R, López V. Impacto de las enfermedades laborales en España. Informe. Madrid: Instituto Sindical de Trabajo, Ambiente y Salud; 2007 Feb.

15. Johnson DW, Hammond RJ, SHERman RE. Hearing inan ambulance paramedic population. Ann Emerg Med. 1980; 9: 557-561.

16. Fernández AR, Crawford JM, Studnek JR, WiLkins JR. Hearing problems among a cohort of nationally certified EMS professionals. Am J Ind Med 2010; 53: 264-275.

17. Price TG, Goldsmith LJ. Changes in hearing acuity in ambulance personnel. Prehosp Emerg Care 1998; 2: 308-311.

18. Ayuntamiento de Bilbao. Ordenanza Municipal de Protección del Medio Ambiente. 2000. Citado 23 marzo 2012. Disponible en: http:// www.bilbao.net/castella/mambiente/normativa/coma0000.pdf

19. Ayuntamiento de Madrid. Ordenanza de Protección de la Atmósfera contra la Contaminación por Formas de Energía. 2004. Citado 23 marzo 2012. Disponible en: http://www.madrid.org/bdccm/normativa/PDF/Ruidos\%20 y\%20vibraciones/Compilacion/CPRUID.pdf

20. Ayuntamiento de Huesca. Ordenanza Municipal reguladora de la emisión y recepción de ruidos y vibraciones del Ayuntamiento de Huesca. 2003. Citado 23 marzo 2012. Disponible en: https://sede.huesca.es/_archivos/ficheros/ordenanzas/ordenanzas-no-fiscales/ ordenanza_ruidos.pdf

21. Ayuntamiento de A Coruña. Ordenanza Municipal medioambiental reguladora de la emisión y recepción de ruidos y vibraciones y del ejercicio de las actividades sometidas a licencia. 1997. Citado 23 marzo 2012. Disponible en: http://www.coruna.es/servlet/ Satellite pagename $=$ Urbanismo $/$ Page $/$ Urbanismo-Generica\&cid=1207120309099\&argIdi oma $=$ es\&itemID=900102285086588046\&item Type=Contenido 